Vol. 4, Special Issue 2, January 2017

\title{
Synchronization of Three Phase with Electrical Grid-A Survey
}

\author{
Lade Shweta ${ }^{1}$, Kadam S.S \\ Student E\&TC Department, Dr. Daulatrao Aher College of Engineering, Karad, India ${ }^{1}$ \\ Assistant Prof. E\&TC Department, Dr. Daulatrao Aher College of Engineering, Karad, India ${ }^{2}$
}

\begin{abstract}
In order to synchronizing the parameters of inverter and grid system, control algorithm plays an important role. Control algorithm used to generate PWM pulses. The total harmonic distortion is generated by non-linear loads. The various methods are available for synchronization and reducing total harmonic distortion. One of them SPWM method, it is reliable and easy method and also MATLAB Simulink is used for proposed.
\end{abstract}

Keywords: synchronization, grid, inverter, THD (total harmonic distortion), control algorithm, PWM pulses.

\section{INTRODUCTION}

The use for electricity for commercial, domestic loads in industrial and rural, urban and semi-urban area has developed year by year. Demand on renewable energy increased by the energy consumption and maximum grid connected systems is used. If this things are used in large scale then output current cannot be vanished its harmonic pollution. According to this standard the inverter output is harmonically distorted than output of grid. The isolating transformer is connected by many distributed resources via grid resources. The power supply use to require in the generated voltages to elimination of dc component for increasing the protection. To reduce the undesirable harmonic content of output current transformer this fact can be used as an advantage. Especially in high power application power quality of grid connected inverter is an important factor. By using voltage quality, the power quality is determined when voltage is controlled variable. In order to provide the required load voltage, inverter system works in standby mode or grid connected mode. In load scheduling condition, the inverters work in standby mode and provide the required power to the load. The power available through renewable systems is in DC form, inverters are preferred instead of alternators. Voltage, frequency and phase are the parameters of the inverter can be controlled for the purpose of synchronization with the related parameters of the grid system. Synchronization of inverter parameters like Voltage, frequency and phase with grid systems can be possible by specific control algorithm. The output from the inverter system can be varied with synchronization of grid system to meet the load requirement.

\section{PROPOSED WORK}

The basic requirement of the system is match the parameters (phase, amplitude, frequency, voltage and current) of inverter and grid. When grid is in offline mode or load shading mode at that time inverter is in standby mode.

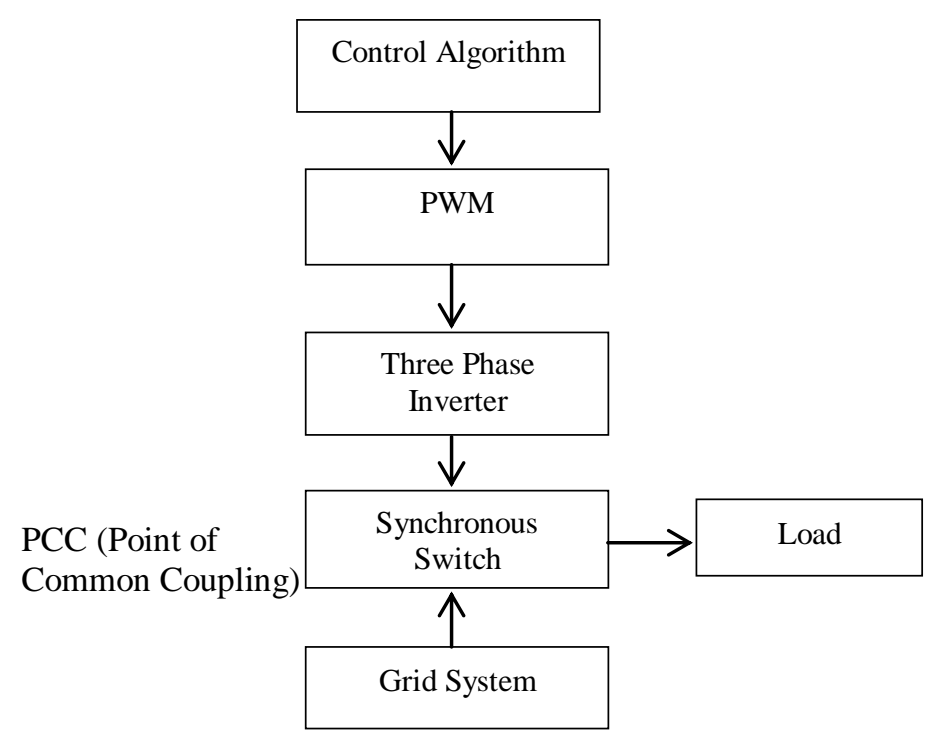

Fig.1.Block Diagram

By using control algorithm we generate the PWM waveform for trigger inverter. The shape of PWM is sinusoidal type used. The PWM is gives to the six switches used in inverter to get output.

PCC is nothing but point of common coupling; it is used as synchronous switch between grid and inverter. It is used for matching the parameters of grid and inverter. Signals of inverter from upward side and signals of grid system from downward side are given to the synchronous switch (PCC).

Basically strength of inverter signals are weak as compare to signals of grid system, therefore synchronous switch increase the strength of inverter signals. These signals strength approximately matched with signals of grid system finally output of synchronous switch is given to load. When grid is in offline mode, the load is work on inverter. 


\section{LITERATURE REVIEW}

In this strategy utilizes common mode signal circuit is use in parallel system as channel of communication between individual inverters. One inverter module as source and synchronous signal is send to all other modules through common mode current .each inverter module will receive and transform signal used as synchronize voltage command for individual inverter. This approaches enables synchronization in the parallel multi-inverter system there is no effect of any output voltage variation or any load change [1].

The algorithm is use to improve in high power application, where switching frequency is very low. This is achieved by maintaining synchronization of half wave symmetry, quarter wave symmetry in PWM waveforms. The principle of operations achieved by using (SVPWM) space vector PWM this algorithm is verified experimentally. The waveform of inverter output voltage for different operating condition of drive present the performance measure in terms of (THD) total harmonic distortion. This THD is calculated for linear modulation region of drive [2].

The use of the controller area network communication protocol for H-bridge multilevel (HBML) inverters, and pulse width modulation generation and voltage regulation is represent in this paper. In that, it will show performance and reliability of distributed controllers for HBML inverters. It can be improved with less communication hardware requirements, when using the represented method. It is also shown that the expansion and modularization capability of the HBML inverter. The proposed hardware is described in detail and the validity of the proposed system is verified[3].

This paper gives a new configuration for modular inverter systems, mainly input-series output-series (ISOS) connected inverter systems, which are very applicable for high-input voltage and high-output-voltage applications. The actual aim of this system to achieve input voltage sharing (IVS) and output voltage sharing (OVS) among the respective modules. The IVS and OVS can be simultaneously achieved only by the application of a compound strategy; It involves controlling not only IVS but also amplitudes or phases of the modules' output voltages. Then, a practical implementation of the compound strategy, which combines a method of IVS with synchronization of the output phase angles [4].

In order to fulfill our requirement for grid interconnection to control distributed power generation system(DPGS).for this purpose grid synchronization algorithm perform important role in DPGS. Also we study in this paper one of the synchronization strategy that use (PLL) phases locked loop \& its various types. The main application of this synchronization method is for distributed generation units with renewable energy sources, which uses different types of power converter and synchronization is represent in the form of voltage, frequency and phase angle of voltage of system[5].
Grid connected solar system use converter circuits uses two: DC to DC booster and PWM inverter. This combination of converter decrement quality and efficiency of electric power. The fast variation of solar radiation is emanated by digital proportional integral controllers and this inverter gives less total harmonic distortion [6].

The shifted space vector pulse width modulation (SVPWM) method is used to control dc-link resonant inverter is presented. As the traditional SVPWM, the shifted SVPWM has same effective vector and operation time in all six space sectors while sequence of vectors change according to current flow direction. To increases the switching frequency of inverter, reducing switching frequency of dc-link resonant circuit and improve the utility of dc-link voltage are main aims of this method. The possibility of the shifted SVPWM approach verified by simulation and experimental result [7].

In high power applications, in order to reduce the harmonics the output voltage of voltage source inverter. (VSI) must be synchronized with its fundamental component. It is possible to obtain synchronization with proper selection of switching states. This paper introduce the SVPWM algorithm used in over modulation region which gives guarantees of synchronization, half wave symmetry, quarter wave symmetry and three phase symmetry. The result of this proposed algorithm compared with conventional algorithm and it gives better performance of VSI [8].

The harmonic current and voltage generated in power system by nonlinear loads. In this paper use the multiple filters with nonlinear loads for investigate effect of inverter output. The simulation results presents under nonlinear loads to verify proposed system [9].

The three phase DC-AC inverter for grid connected PV system using third harmonic injection PWM (THIPWM) technique. The third harmonic injection technique for (PWM) is employed to reduce total harmonic distortion because the SPWM technique is easiest modulation scheme to understand but required complicated mathematical operation. Therefore the THIPWM make inverter suitable for grid connection matching inverter voltage with grid voltage. The MATLAB Simulink is used for proposed system [10].

The control technique consist of dead beat line voltage natural frame current controller, adaptive neural network (NN) based disturbance estimator and energetic sensor less synchronization loop. The approximated dynamics provides necessary energy shaping in the inverter control voltage to emaciated grid voltage interference. The feature of NN algorithm allows easy adaption design at different grid disturbance and operating condition [11].

The grid connected photovoltaic system feed electricity directly to electrical network operating parallel to the conventional source. The system deals with design and simulation of three phase inverter in MATLAB and Simulink. It can be part of photovoltaic grid connected system. Also this system represent control algorithm for three phase grid connected photovoltaic system in that 
inverter design for grid connected photovoltaic array can of issues related to this paper. We express our warm matching output of sinusoidal current and grid thanks to Ms. Supriya S. Kadam For her support and voltage[12].

In this control strategy represents control for three phase three level neutral point clamped inverter (NPC) for grid connected photovoltaic (PV) system. The maximum power point tracking (MPPT) is capable of providing incremented power from PV array connected to each DClink voltage level. The MPPT system connected with DC link controller so that DC-DC converter is not needed and output shows accurate and quick response [13].

From parallel inverter system with failure isolation and hot swap features control the output voltage regulation and synchronizing parameter of inverter. This system providing identical and different power rating with help of failure isolation feature and this feature also use to scanning the circuit. This system represent power capacity can be readily expanded because of that inverter failure disturbance can be reduced [14].

A synchronverter is a type of inverter which behaves like synchronous generator which provides power system to controlling grid connected renewable energy. In this, most important step is to improve synchronverter as selfsynchronverter by removing synchronization units and also it is self- synchronized before grid connection and tracking the frequency after grid connection because of that improve the performance of system reducing complexity of controller[15].

In the multilayer control system no of control layers are used to perform parallel operation without any intercommunication. The first layer is an improved subsidence method that represent power terms into conventional subsidence scheme. The second layer is use to immunize voltage deviation caused by subsidence control method. The third layer use for adjusting angle of inverter which is nearest to common AC bus [16].

\section{IV.CONCLUSION}

The inverter can operate in landed and grid connected mode. When fault occur grid need to operate independently from grid to supply without any interference power to the load. The control algorithm used to reduce harmonic distortion to give Minimum error output to improve performance of grid. The PCC used for synchronization of inverter with electrical grid to allow synchronization between grid parameter \& inverter parameter such as voltage, phase, frequency. control algorithm used to generate PWM pulses on the bases of Synchronization.

\section{ACKNOWLEDGMENT}

We are using this opportunity to express my gratitude to everyone who supported me for writing this review paper. We are thankful for their guidance and invaluably advice during this work. We are sincerely grateful to them for sharing their truthful and illuminating views on a number guidance at Department of Electronics and Telecommunication Engineering; Daulatrao Aher College of Engineering, Karad, 415110; Maharashtra, India

\section{REFERENCES}

[1] Yeong Jia Cheng, Eng Kian. Kenneth SNG, “A Novel communication strategy for decentrilised control of paralleled multi-inverter system", IEEE transaction on power electronics, vol_21, no.1.

[2] Abdul Rahiman Beig, G.Narayanan, V.T.Rangnathan, "Modified SVPWM algorithm for three level VSI synchronized \& symmetrical waveforms" IEEE transaction on industrial electronics, vol_54,no.1.

[3] Young-Min Park, Ji-Yoon.Yoo, Sang_Bin Lee "practical implementation of PWM synchronization \& phase shift method for cascaded H-bridge multilevel inverter based on standard serial communication protocol" IEEE transaction on industry applications, vol_44,no.2.

[4] Tinzhi Fang, Xinbo Ruan, chik. Tse, Fellow, "control strategy to achieve input \& output voltage sharing for input series-output series-connected inverter system "'IEEE transaction on power electronics,vol_25,no.6.

[5] Rajan J.Devi, Supriya S.Kadam, "phase locked loop for synchronization of inverter with electrical grid ',international journal of engineering research \& technology(IJERT) vol_4 issue 02 .

[6] K N S Durga prakash, ch Narendra kumar "grid connected thirteen level inverter for PV system using PI controller', international journal of engineering research \& technology(IJERT)vol_1 issue 5 .

[7] Sanbo Pan, Junmin Pan, Zuohua Tian, "A shifted SVPWM method to control DC-link resonant inverter \& its FPGA realization' 'IEEE transaction on industrial electronics, vol_59,no.9.

[8] Abdul Rahiman Beig, "synchronized SVPWM algorithm for over modulation region of low swithing frequency medium-voltage three-level VSI''IEEE transaction on industrial electronics,vol_59,no.12, December 2012.

[9] Jawad Faiz \& Ghazanfar Shahgholian "moduling \& simulation of a three phase inverter with rectifier type nonlinear loads" Armenian Journal of Physics,2009,vol_2, issue 4,September 2009.

[10] Anita \& Prerna Gaur "simulation of high efficiency grid connected THIPWM-three phase PV inverter" European journal of advances in engineering \& technology

[11] Yasser Abdel-Rady I. Mohamed, Ehab F.EI-Saadany "A robust natural-frame-based interfacing scheme for grid-connected distributed generation inverters" IEEE transaction on energy conversion, vol_26, no.3, September 2011.

[12] Alatza Y.Abdelaziz, Ahmed M. Atallah \& Raihan S.Jumaah "modeling \& simulation of three phase two stage grid connected photovoltaic system", December 2013.

[13] Serkan Sezen, Engin Ozdemir "modeling Simulation \&control of three level multilevel inverter for grid connected photovoltaic system" journal of optoelectronics \&advanced materials, vol_15, no.3, march\April 2013.

[14] Tsai-Fu Wu, Hui-Ming Hsieh, Yu-En Wu \& Yu- llel inverter system with failure isolation and hot -swap features" ,IEEE transaction on applicationvol_43,no.5,September/October 2007.

[15] Quing-Change Zhong, Phi-Long Nguyen, Zhenyu Ma, Wanxing Sheng, "Self-synchronized synchronverter: inverters without a dedicated synchronization unit", IEEE transaction on power electronics, vol_29, no.2, February 2014.

[16] Ming Hua, Haibing Hu, Yan Xing, Josep M.Guerrero "Multilayer control for inverter in parallel operation without intercommunication", IEEE transaction on power electronics, vol_27, no.8, August 2012. 